\title{
Simultaneous Implementation of Experimental Centric Pedagogy in 13 ECE Programs
}

\section{Prof. Kenneth A Connor, Rensselaer Polytechnic Institute}

Kenneth Connor is a professor in the Department of Electrical, Computer, and Systems Engineering (ECSE) where he teaches courses on electromagnetics, electronics and instrumentation, plasma physics, electric power, and general engineering. His research involves plasma physics, electromagnetics, photonics, biomedical sensors, engineering education, diversity in the engineering workforce, and technology enhanced learning. He learned problem solving from his father (ran a gray iron foundry), his mother (a nurse) and grandparents (dairy farmers). He has had the great good fortune to always work with amazing people, most recently professors teaching circuits and electronics from 13 HBCU ECE programs and the faculty, staff and students of the SMART LIGHTING ERC, where he is Education Director. He was ECSE Department Head from 2001 to 2008 and served on the board of the ECE Department Heads Association from 2003 to 2008.

Dr. Yacob Astatke, Morgan State University

Dr. Charles J. Kim, Howard University

Charles Kim is a professor in Electrical and Computer Engineering at Howard University. He received a Ph.D. degree in Electrical Engineering from Texas A\&M University in 1989, and worked as a researcher at Texas A\&M University before he took an assistant professor at the University of Suwon in 1994. Since 1999, he is with Howard University. Dr. Kim's research interests include energy systems, fault detection and anticipation, embedded computing, safety-critical computer systems, and intelligent systems application. Dr. Kim is active in practicing experiential learning in engineering education with personal instrumentation such as mobile studio.

Dr. Abdelnasser A Eldek, Jackson State University

Dr. Abdelnasser A. Eldek obtained his Ph.D. in Electrical Engineering in 2004 from the University of Mississippi. Currently, he is Associate Professor with the Department of Electrical and Computer Engineering at Jackson State University. His main research areas include Applied Electromagnetics, Antennas, Phased Arrays, RF/Microwave Circuits, Metamaterial, and Numerical Methods.

\section{Dr. Hamid R. Majlesein, Southern University and A\&M College}

Dr. Majlesein's is currently a professor at the Department of Electrical Engineering in Southern University and A\&M College. He also has worked as the Department Head of Electrical Engineering from 2010-2014. His research interests are in the areas of Electric Power Systems, Computer Networks, and Digital Signal Processing. Dr. Majlesein's teaching interests are in the areas of Circuits Analysis, Electric Machinery, Signals and Systems, Digital Signal Processing, Control Systems, Power Systems, Probability and Random Signals, and Computer Networks.

\section{Prof. Petru Andrei, Florida A\&M University \& Florida State University}

Dr. Petru Andrei is Associate Professor and Graduate Program Director in the Department of Electrical and Computer Engineering at the Florida A\&M University and Florida Stat University (FAMU-FSU) College of Engineering. He is the FSU campus education director for the NSF-ERC Future Renewable Electric Energy Delivery and Management Systems Center (FREEDM) and has much experience in recruiting and advising graduate, undergraduate, REU, and K-12 students, as well as in working with RET teachers. Dr. Andrei has published over 100 articles in computational electronics, electromagnetics, energy storage devices, and large scale systems.

Dr. John Okyere Attia P.E., Prairie View A\&M University

Kathy Ann Gullie PhD, University at Albany/SUNY

(C)American Society for Engineering Education, 2015 
Dr. Kathy Gullie has extensive experience as a Senior Evaluator and Research Associate through the Evaluation Consortium at the University at Albany/SUNY. She is currently the principal investigator in several educational grants including an NSF engineering grant supporting Historically Black University and Colleges; "Building Learning Communities to Improve Student Achievement: Albany City School District", and "Educational Leadership Program Enhancement Project at Syracuse University" Teacher Leadership Quality Program. She is also the PI on both "Syracuse City School District Title II B Mathematics and Science Partnership: Science Project and Mathematics MSP Grant initiatives.

\section{Dr. Corey A Graves, North Carolina A\&T State University}

Corey A. Graves is an associate professor and the director of the Auto Mobile Pervasive and Embedded Design 9AMPED) Laboratory in the Electrical and Computer Engineering Department at North Carolina A\&T State University. His research interests include developing pervasive computing systems for education enhancement as well as health-related application. Graves has a $\mathrm{PhD}$ in Computer Engineering from North Carolina State University. Contact him at cag@ncat.edu

\section{Dr. Ali Reza Osareh, NC A\&T State University}

Ali Osareh received his $\mathrm{PhD}$ from Virginia tech in 1994. He has worked in the industry including wireless design before joining the Department of Electrical and Computer Engineering at North Carolina Agricultural and Technical State University in 2000. He is specializing in Energy and Power Systems, Industrial Automation and Control system. As part of HBCU-ECP project he teaches EE and non-EE students how to utilize the board for in class experiments and other design projects. He is also currently doing a collaborative research with a local industry in smart grid. Dr. Osareh can be reached at osareh@ncat.edu 


\section{Collaborative Research: Center for Mobile Hands-on STEM}

Remarkable progress has been made in the development and implementation of hands-on learning in STEM education. The mantra of See One, Do One, Teach One overly simplifies the idea but does provide a helpful structure to understand how many engineering educators are attempting to change the learning experience of our students. Until recently, this effort has been faced with a major limitation. We can easily incorporate traditional paper and pencil and numerical analysis, synthesis, and simulation in our classrooms. However, the remaining key aspect of doing the job of an engineer - experimentation - has only been included through the use of expensive and limited-access lab facilities. Small, low-cost Mobile Hands-On STEM (MHOS) learning platforms (e.g., myDAQ, Analog Discovery, and Circuit Gear Mini) provide almost unlimited opportunities to solve this remaining problem in engineering courses. Pedagogy based on these tools has been implemented and studied in several institutions in the US and in other countries, impacting thousands of students each year. In all cases in which hands-on learning has been studied, the pedagogy has been successfully implemented. This has occurred even in traditionally theory-only based courses, resulting in more engaged students and instructors. Although the initial assessments of this new approach to STEM education argue for broad application, the definitive case for its adoption has yet to be documented so that all STEM educators can fully appreciate its merit.

The Center for Mobile Hands-On STEM is pursuing activities that support the following goals:

- Gather strong evidence of the effectiveness of Mobile Hands-On STEM (MHOS) pedagogy on student learning.

- Develop an effective and pro-active dissemination strategy for the entire STEM educational community.

To achieve these goals, we have recently focused on:

- Creating and implementing new standardized assessment tools that measure student learning, especially through the development of new experimentally focused concept inventories, as well as measure ease of adoption by instructors.

- Identifying implementation barriers for wide-spread adoption and how these might be overcome by applying the business start-up methodology of the NSF I-Corps program, working with faculty who have recently received funding to implement the mobile pedagogy, and holding focus groups among different constituencies.

Both of these general areas of activity represent works-in-progress. In the former we are investigating formulations of concepts and possible learning and assessment activities and collecting data on their effectiveness. We identify three objectives of Hands-On instruction, 1) to apply instrumentation to make measurements of physical quantities, 2) to identify limitations of models to predict of real-world behavior, and 3) to develop an experimental approach to characterize and explain the world. We have consulted with experts to develop a list of common misconceptions students display in laboratory instruction. A unique feature in testing Hands-On 
concepts is that laboratory skills are inextricably tied to analytical concepts and therefore both analytical and hands-on concepts have to be tested in order to distinguish the root cause of the misunderstanding. Based on these common misconceptions, test questions are being developed and data is being collected on their effectiveness to assess learning. Feedback from faculty and students interested in MOHS pedagogy is being solicited. For the latter, we have had a group of our colleagues go through I-Corps training as part of a pilot program to determine whether the ICorps model could be used to expand the impact of educational research. In addition, strong collaborative relationships have been developed with new groups who are aggressively implementing similar pedagogy throughout all of their engineering programs.

\section{Implementation Outside of ECE: Mechanical Engineering}

In order to expand the mobile hands-on learning methodology into mechanical engineering, two experiments were developed and tested in a rigid-body dynamics class. The criterion for selection of experiments was (1) What do students have most difficulty in learning? (2) What new technologies exist that can be leveraged to create small portable experiments? (3) What hands-on activities would be most effective in learning and retaining new concepts? (4) How can the measurements be quantified so that comparisons can be made between theoretical predictions and experimental results? Because of the difficulties in instrumenting a body in motion, the initial versions of the experiments were done as a demo by the instructor in front of class. In each case, the data from the experiments was transmitted to the students for them to analyze during the same class period. In this way, it was a cross between traditional instructor demos and student run hands-on experiments.

One of the experiments examined centripetal acceleration of a car running on a semicircular path. The accelerations were measured in two ways: with an accelerometer on a microcontroller or a phone on the cart, and by a vision tracking software that analyzed a video of the motion of the cart. The other experiment was to analyze rolling contact. For planar motion, the confusion centers on two concepts: (1) the contact point between the disk and a stationary surface has instantaneously zero velocity; i.e., it is an instant center of velocity, (2) the point of contact does not have zero acceleration. In the first case, students have a difficult time believing that the contact point can be momentarily at rest. Once they see that, they have trouble reconciling that the contact point has zero velocity but has high acceleration away from the contact plane. A disk with a red dot marked on the rim was video-taped as it rolled. Students used video processing software to trace the 2-dimentional motions of that point during rolling.

Several performance assessments were conducted to determine the ability of students to learn from the rolling contact experiment: two related basic concepts quizzes and a related question on the final exam concerning the acceleration of the contact point on a wheel undergoing threedimensional rolling. No students had conceptual errors on the final exam. These experiments will undergo further refinement based on these pilot tests. 


\section{New MOOC Implementation: Electronics}

A new MOOC was developed on electronics that contained video demonstrations of several hands-on experiments using a myDAQ as well as several optional labs that people may do on their own. Over 26,000 were enrolled in the course. Adding to the 55,000 people who were enrolled in a similar MOOC on Linear Circuits, the exposure to mobile hands-on learning with circuits and electronics experiments was considerable. These MOOCs were used to teach a distance learning portion of a regular on-campus course for credit. In addition to the MOOC videos and homework, those distance learning students were required to take tests and do the labs (the labs were only optional for the public MOOC students). The discussion forum was essential for the distance learning students, who were located all around the world. They did not have lab partners and did the labs individually at their homes with their own equipment. They sought help in the forums by posting images of their breadboards asking for help in troubleshooting the wiring and posted screen shots of the software instrument panels asking if they used the correct settings. Other students responded with comments and answers.

\section{I-Corps-L}

In January and February 2014, NSF funded a pilot program to determine whether their I-Corps methodology used to facilitate the commercialization of ideas from technical research could also be applied to engineering educational research. Three representatives from the MOHS project were selected as one of 9 teams and given approximately $\$ 50 \mathrm{k}$ of supplemental funding. I-Corps is an intensive, almost bootcamp experience in which a team of 3 is thoroughly trained in developing a new business based on the Business Model Canvas (Lean Launchpad from Stanford and Berkeley). The process begins and ends with a multi-day workshop (in DC), followed by weekly 2 hour video conferences which include additional training and reporting on our efforts to develop our plan for spreading the use of our educational ideas (in our case Mobile Hands-On Learning). Included in the process is a requirement to test out our hypotheses (e.g. our value proposition, possible income streams ...) through a minimum of 100 customer interviews. The process ran throughout January and February and was nearly a full-time effort. After February, we have continued to work on the plan we developed (to create a new division at ASEE to bring some structure and support to MOHS pedagogy). There was also a one day workshop at ASEE in which the 9 pilot groups presented to help educate and recruit the next cohorts. Based on the success of the pilot, the decision was made to expand I-Corps to include learning. In addition to helping us clarify our plans for disseminating MOHS, we also were able to present our story several times to the other eight teams, all of whom are active, productive leaders in engineering education research.

\section{Supporting Other Groups Implementing Related Pedagogy}

We continue to expand our network of people doing MOHS relevant work and are presently nurturing new programs with community colleges, high schools, industry and NSF Engineering 
Research Centers. From these efforts, we have recently seen the creation of two new programs that have great potential to impact the diversity of the engineering workforce.

Experimental Centric Based Engineering Curriculum for HBCUs: The goal of this 3 year, NSF funded program is to create a sustainable Network of engineering faculty at Historically Black Colleges and Universities to focus on the development, implementation, and expansion of an experiment-centric instructional pedagogy, based on the Mobile Studio. The project is implementing this pedagogy across the 13 HBCUs participating in the network and studying the effect of the implementation on motivation and retention. The 13 schools are Howard, Morgan

State, Jackson State, Hampton, Maryland Eastern Shore, Florida A\&M, Southern, Tuskegee, North Carolina A\&T, Tennessee State, Alabama A\&M, Prairie View A\&M, and Norfolk State. The leadership for this project comes primarily from MOHS program participants from Howard, Morgan State and Rensselaer. Faculty participants from 11 of the 13 schools have had little or no experience with MOHS-style pedagogy. To assist them in developing new course materials, workshops were held at Howard in December 2013 and July 2014. Both workshops included major contributions from the leadership team noted above and the MOHS program leader from Georgia Tech. In addition, we facilitate connections with faculty at other institutions doing relevant work and with organizations that can provide additional funding. Excellent overall progress is being made by nearly all of the teams on first year intro to engineering and intro to ECE courses, circuits and electronics courses, undergraduate research and senior design.

\section{Diffusion of Mobile Hands-on Learning in Puerto Rico Using the Analog Discovery Board:}

This program is inspired by the HBCU ECP project and involves two NSF funded workshops in 2015 (February and September) that will bring together all faculty teaching circuits and electronics courses at universities in Puerto Rico to begin the process of spreading mobile handson learning and make preparations for a larger NSF grant to fully realize the potential of handson learning in engineering education. Two members of the MOHS team will lead the two workshops.

\section{ECE Leadership Activities}

MOHS participants have maintained an active presence at the annual ECE Department Heads Association (ECEDHA) meeting to keep department heads and chairs up-to-date on the rapidly changing world of MOHS pedagogy. Most recently, one of the I-Corps-L team attended the meeting in Napa, CA as a continuation of the process of obtaining feedback on our plans from potential 'customers,' using the terminology learned during training. He also attended diversity sessions to support the HBCU ECP group. 